\title{
REPELLENT ACTIVITY OF DEVERRA TRIRADIATA (APIACEAE) EXTRACTS AGAINST ANOPHELES SERGENTII THEOBALD, CULEX PIPIENS LISTON AND CULEX ANTENNATUS BECKER MOSQUITOES
} By

\author{
AHMED Z. I. SHEHATA \\ Department of Zoology, Faculty of Science, Al-Azhar University, Nasr City, Cairo, \\ Egypt (Correspondence:ahmedzeinhom00@gmail.com)
}

Abstract

The present study evaluated the repellent activity of hexane, chloroform, methanol and ethyl acetate extracts from Deverra triradiata aerial parts against three mosquito species (Anopheles sergentii, $\mathrm{Cu}$ lex pipiens and Culex antennatus). At 3.33, 1.67, $0.83 \& 0.42 \mathrm{mg} / \mathrm{cm}^{2}$, all tested extracts showed a variable degree of repellency against tested mosquito species depending on solvent used in extraction. The highest repellent activity attained by hexane extract, with $\mathrm{RD}_{50}$ equal to $0.704,1.122 \& 0.92$ $\mathrm{mg} / \mathrm{cm}^{2}$ against $A n$. sergentii, $C x$. pipiens and $C x$. antennatus starved females, followed by ethyl acetate $\left(0.904,1.323 \& 0.9 \mathrm{mg} / \mathrm{cm}^{2}\right)$, chloroform $\left(1.101,1.367 \& 1.157 \mathrm{mg} / \mathrm{cm}^{2}\right)$ and methanol $(1.183$, $1.578 \& 1.323 \mathrm{mg} / \mathrm{cm}^{2}$ ) extracts. Also, $\mathrm{RD}_{90}$ of hexane, chloroform, methanol and ethyl acetate extracts recorded $2.567,2.92,3.067$ and $2.88 \mathrm{mg} / \mathrm{cm}^{2}$ against An. sergentii, 3.027, 3.317, 3.593 \& $3.547 \mathrm{mg} / \mathrm{cm}^{2}$ against $C x$. pipiens, $2.703,3.09,3.267 \& 2.81 \mathrm{mg} / \mathrm{cm}^{2}$ against $C x$. antennatus starved females, respectively. In addition, complete repellency time was varied according to solvent used in extraction. D. triradiata tested extracts showed a strong biting deterrency against tested mosquito species, where the highest complete repellency time $(187.7 \mathrm{~min})$ a achieved by methanol extract against An. sergentii starved females at $3.33 \mathrm{mg} / \mathrm{cm}^{2}$ and the lowest complete repellency time $(57.7 \mathrm{~min}) \mathrm{rec}-$ orded by hexane extract against $C x$. pipiens bites at $0.42 \mathrm{mg} / \mathrm{cm}^{2}$, respectively.

Keys words: Anopheles sergentii, Culex pipiens, Cx. antennatus, repellency, Deverra triradiata.

\section{Introduction}

Mosquito bites causes allergic responses including local skeeter syndrome such as urticaria and angioedema (Abdel-Motagaly et $a l, 2017$ ), and transmission of many diseases as malaria, lymphatic filariasis, dengue, Japanese encephalitis with annual millions of deaths (El-Bahnasawy et al, 2013). Huge numbers of mosquitoes were reported in Egypt (Mikhail et al, 2009). Malaria is still the important cause of infectious disease mortality in many parts of Africa, and some areas in Asia and Latin America (WHO, 2014). Egyptian cases of dengue fever and Aedes aegypti were reported (Morsy, 2018). Also, $C x$. antennatus (Becker) was the vector of Rift Valley Fever virus during an outbreak in the Nile Delta of Egypt (Hanafi et al, 2011). Personal protection products, including repellents, are widely used to reduce the transmission of diseases by minimizing the contact between humans and vectors (Pitasawat et al, 2003). Commercial repellent products contain che-mical compounds as DEET (N, N-diethyl-3-methylbenzanmide), showed best repellency against mosquitoes (Walker et al, 1996). The side effects of these chemical products varied from mild to fatal (Qiu et al, 1998) which stimulated to get repellents derived from medicinal plants and herbs to alternate the DEET (Tawatsin et al, 2001). Deverra triradiata belongs to Apiaceae is a medicinal plant found in south Sinai, Egypt and locally used for get rid of dyspnea.

This study aimed to offer an opportunity for developing alternatives to rather expensive and environmentally hazardous organic insecticides.

\section{Materials and Methods}

Collection and rearing of mosquitoes: Larvae of Anopheles sergentii and Culex pipiens were collected from El-Fayoum Governorate, in March 2017, while Cx. antennatus larvae collected from Shubramunt, Giza in April 2017. All mosquitoes were kept for several generations, Medical Insectary, Animal House, Department of Zoology, Al-Azhar Faculty of Science; under controlled temper- 
atue of $\left(27 \pm 2^{\circ} \mathrm{C}\right), \mathrm{RH}(70 \pm 10 \%)$, and light and dark cycles (12-12). Larvae were provided with finely ground dog biscuit and adults were fed on $10 \%$ sucrose solution and were periodically allowed to take a blood meal from the pigeon (Haldar et al, 2014).

Plant collection and preparation of crude extract: Deverra triradiata was collected in April 2017 from South Sinai Governorate, away from sun rays, left to dry at room temperature $\left(25-30^{\circ} \mathrm{C}\right)$ for 5 to 10 days and pulverized to powder commercial electrical stainless steel blender. Extraction was performed using hexane, chloroform, methanol and ethyl acetate (El-Sheikh et al, 2016).

Repellent activity: For repellent activity of D. triradiata extracts, cages $(60 \times 60 \times 60 \mathrm{~cm})$ were used. Different weights from each extract were dissolved in $2 \mathrm{ml}$ of the solvent with a drop of Tween 80 separately in glass $4 \times 4 \mathrm{~cm}$ to prepare different concentrations. After removing feathers from the pigeon abdomen, each concentration was directly applied onto $5 \times 6 \mathrm{~cm}$ of ventral surface to evaluate the repellency against $A n$. sergentii, $C x$. pipiens \& $C x$. antennatus. After 10min., the pigeons were placed in cages containing one hundred An. sergentii, Cx. pipiens \& $C x$. antennatus starved females (5-7d-old) for three hours. Control tests were carried out alongside with the treatments using hexane, chloroform, methanol \& ethyl acetate with a drop of Tween 80 separately. Each test was repeated three times to get a mean value of repellent activity. The time in which mosquitoes began to descend on the pigeon for feeding has been recorded. After treatments, fed and unfed females were calculated (Abbott, 1925): Repellency $\%=[\% \mathrm{~A}-\% \mathrm{~B} / 100-$ $\% \mathrm{~B}] \times 100(\mathrm{~A}=$ unfed treatment females $\%$ and $\mathrm{B}=$ unfed females control\%. Statistical analysis: Data were tabulated and analyzed using Statistical Package Social Science software version 11.5 (SPSS, 2007).

\section{Results}

The maximum repellent activity was observed for Deverra triradiata hexane extract against tested mosquito species as compared with other extracts. At the highest concentration $\left(3.33 \mathrm{mg} / \mathrm{cm}^{2}\right)$ hexane extract recorded 91.8, 85.0 and $90.4 \%$ repellent activity against Ano. sergentii, $C x$. pipiens and $C x$. antennatus starved females, respectively.

The highest repellent activities achieved by chloroform, methanol and ethyl acetate extracts against $A n$. sergentii were 86.8, 85.9 and $90.7 \%$ at $3.33 \mathrm{mg} / \mathrm{cm}^{2}$, respectively. Also, hexane extract at the lowest dose $\left(0.42 \mathrm{mg} / \mathrm{cm}^{2}\right)$ provided complete protection against $A n$. sergentii bites for at least 116.3 min vs. $9.3 \mathrm{~min}$ for the control group, respectively (Tab.1).

D. triradiata chloroform, methanol \& ethyl acetate extracts evoked variable repellent activities against $C x$. pipiens starved females, where, at $3.33,1.67,0.83 \& 0.42 \mathrm{mg} / \mathrm{cm}^{2}$ the repellent activities were 80.0, 68.5, 53.6, $35.6 \%$ for chloroform extract; 76.7, 60.6, $46.9,32.8 \%$ for methanol extract and 80.5, $74.2,49.1,38.3 \%$ for ethyl acetate extract, respectively. D. triradiata hexane, chloroform, methanol \& ethyl acetate extracts provided highest protection against $C x$. pipiens bites $(105.0,117.0,136.0 \& 125.7 \mathrm{~min})$ at highest dose $\left(3.33 \mathrm{mg} / \mathrm{cm}^{2}\right)$, respectively (Tab. 2).

The highest and lowest repellent percentages recorded against $C x$. antennatus starved females were $83.2 \& 39.0$ by chloroform extract; $82.7 \& 38.1$ by methanol extract; 89.0 \& 56.4 by ethyl acetate extract at 3.33 \& $0.42 \mathrm{mg} / \mathrm{cm}^{2}$, respectively at doses of 3.33 , $1.67,0.83 \& 0.42 \mathrm{mg} / \mathrm{cm}^{2}$, the complete protection times against $C x$. antennatus bites recorded by hexane and chloroform extracts were 158.0, 146.3, 136.0, $112.7 \& 175.3$, $155.3,141.0,121.7 \mathrm{~min}$, respectively. Complete protection times recorded by methanol and ethyl acetate extracts against $C x$. antennatus bites were 169.0, 157.7, 149.3, 147.0 \& 167.7, 166.7, 158.3, $146.7 \mathrm{~min}$, respectively, compared with $8.3 \& 9.7 \mathrm{~min}$ for untreated ones (Tab. 3). Hexane extract from aerial parts gave the highest repellent activity against females as compared with other extracts, where, $\mathrm{RD}_{50}$ were $0.704,1.122$ \& 
$0.92 \mathrm{mg} / \mathrm{cm}^{2}$ against $A n$. sergentii, Cx. pipie $n s$ and $C x$. antennatus starved females. The lowest repellent activity was by methanol extract against $A n$. sergentii, $C x$. pipiens and $C x$. antennatus females with $\mathrm{RD}_{50}$ were $1.183,1.578 \& 1.387 \mathrm{mg} / \mathrm{cm}^{2}$, respectively. $\mathrm{RD}_{90}$ of hexane, chloroform, methanol and ethyl acetate extracts gave 2.567, 2.92, 3.067 \& $2.88 \mathrm{mg} / \mathrm{cm}^{2}$ against An. sergentii, 3.027, $3.317,3.593 \& 3.547 \mathrm{mg} / \mathrm{cm}^{2}$ against $C x$. pipiens, $2.703,3.09,3.267 \& 2.81 \mathrm{mg} / \mathrm{cm}^{2}$ against $C x$. antennatus starved females, respectively (Tab. 4).

Table 1: Repellent activity of $D$. triradiata different extracts against An. sergentii.

\begin{tabular}{|c|c|c|c|c|c|}
\hline Extract & $\begin{array}{c}\text { Dose } \\
\left(\mathrm{mg} / \mathrm{cm}^{2}\right)\end{array}$ & $\begin{array}{c}\text { Fed females } \\
(\%)\end{array}$ & $\begin{array}{c}\text { Unfed females } \\
(\%)\end{array}$ & $\begin{array}{c}\text { Average repellency } \\
(\%)\end{array}$ & $\begin{array}{c}\text { Complete repellency } \\
\text { time (min.) }\end{array}$ \\
\hline \multirow{5}{*}{ Hexane } & 3.33 & $8.0 \pm 2.6$ & $92.0 \pm 2.6$ & $91.8 \pm 2.5$ & $174.0 \pm 10.4$ \\
\hline & 1.67 & $14.3 \pm 2.1$ & $85.7 \pm 2.1$ & $85.3 \pm 1.7$ & $164.0 \pm 7.9$ \\
\hline & 0.83 & $21.0 \pm 4.0$ & $79.0 \pm 4.0$ & $78.5 \pm 3.6$ & $126.0 \pm 7.0$ \\
\hline & 0.42 & $43.7 \pm 3.8$ & $56.3 \pm 3.8$ & $55.2 \pm 3.2$ & $116.3 \pm 6.5$ \\
\hline & Control & $97.3 \pm 2.1$ & $2.7 \pm 2.1$ & 0.0 & $9.3 \pm 1.5$ \\
\hline \multirow{5}{*}{ Chloroform } & 3.33 & $12.7 \pm 2.5$ & $87.3 \pm 2.5$ & $86.8 \pm 2.8$ & $175.0 \pm 6.0$ \\
\hline & 1.67 & $20.3 \pm 3.1$ & $79.7 \pm 3.1$ & $78.9 \pm 3.0$ & $174.3 \pm 7.2$ \\
\hline & 0.83 & $31.7 \pm 2.9$ & $68.3 \pm 2.9$ & $67.1 \pm 3.8$ & $142.0 \pm 6.6$ \\
\hline & 0.42 & $55.0 \pm 2.6$ & $45.0 \pm 2.6$ & $42.9 \pm 3.2$ & $122.0 \pm 3.0$ \\
\hline & Control & $96.3 \pm 2.5$ & $3.7 \pm 2.5$ & 0.0 & $9.0 \pm 2.0$ \\
\hline \multirow{5}{*}{ Methanol } & 3.33 & $13.7 \pm 1.5$ & $86.3 \pm 1.5$ & $85.9 \pm 1.8$ & $187.7 \pm 6.7$ \\
\hline & 1.67 & $28.3 \pm 2.1$ & $71.7 \pm 2.1$ & $70.7 \pm 1.7$ & $175.7 \pm 3.7$ \\
\hline & 0.83 & $44.3 \pm 3.5$ & $55.7 \pm 3.5$ & $54.1 \pm 3.9$ & $167.3 \pm 8.2$ \\
\hline & 0.42 & $51.3 \pm 1.5$ & $48.7 \pm 1.5$ & $46.9 \pm 1.4$ & $138.7 \pm 5.5$ \\
\hline & Control & $96.7 \pm 1.5$ & $3.3 \pm 1.5$ & 0.0 & $8.7 \pm 1.2$ \\
\hline \multirow{5}{*}{$\begin{array}{c}\text { Ethyl Ace- } \\
\text { tate }\end{array}$} & 3.33 & $9.0 \pm 2.0$ & $91.0 \pm 2.0$ & $90.7 \pm 2.3$ & $179.3 \pm 6.5$ \\
\hline & 1.67 & $30.3 \pm 2.5$ & $69.7 \pm 2.5$ & $68.6 \pm 3.3$ & $174.7 \pm 7.1$ \\
\hline & 0.83 & $31.3 \pm 2.5$ & $68.7 \pm 2.5$ & $67.6 \pm 2.0$ & $164.0 \pm 5.6$ \\
\hline & 0.42 & $40.3 \pm 4.2$ & $59.7 \pm 4.2$ & $58.3 \pm 3.6$ & $150.0 \pm 2.6$ \\
\hline & Control & $96.7 \pm 2.3$ & $3.3 \pm 2.3$ & 0.0 & $9.3 \pm 4.2$ \\
\hline
\end{tabular}

Table 2: Repellent activity of D. triradiata different extracts against $C x$. pipiens.

\begin{tabular}{|c|c|c|c|c|c|}
\hline Extract & $\begin{array}{c}\text { Dose } \\
\left(\mathrm{mg} / \mathrm{cm}^{2}\right)\end{array}$ & $\begin{array}{l}\text { Fed females } \\
(\%)\end{array}$ & $\begin{array}{l}\text { Unfed females } \\
(\%)\end{array}$ & $\begin{array}{c}\text { Average repellency } \\
(\%)\end{array}$ & $\begin{array}{l}\text { Complete repellency time } \\
\text { (min.) }\end{array}$ \\
\hline \multirow{5}{*}{ Hexane } & 3.33 & $14.3 \pm 1.5$ & $85.7 \pm 1.5$ & $85.0 \pm 1.7$ & $105.0 \pm 6.0$ \\
\hline & 1.67 & $26.3 \pm 3.5$ & $73.7 \pm 3.3$ & $72.4 \pm 3.7$ & $92.7 \pm 8.5$ \\
\hline & 0.83 & $34.0 \pm 3.5$ & $66.0 \pm 3.5$ & $64.3 \pm 3.8$ & $75.7 \pm 4.5$ \\
\hline & 0.42 & $55.3 \pm 2.9$ & $44.7 \pm 2.9$ & $41.9 \pm 3.3$ & $57.7 \pm 4.2$ \\
\hline & Control & $95.3 \pm 0.6$ & $4.7 \pm 0.6$ & 0.0 & $6.3 \pm 0.6$ \\
\hline \multirow{5}{*}{ Chloroform } & 3.33 & $19.3 \pm 2.1$ & $80.7 \pm 2.1$ & $80.0 \pm 1.9$ & $117.0 \pm 4.2$ \\
\hline & 1.67 & $30.3 \pm 2.5$ & $69.7 \pm 2.5$ & $68.5 \pm 1.8$ & $94.7 \pm 5.0$ \\
\hline & 0.83 & $44.7 \pm 2.1$ & $55.3 \pm 2.1$ & $53.6 \pm 3.1$ & $89.0 \pm 5.6$ \\
\hline & 0.42 & $62.0 \pm 6.6$ & $38.0 \pm 6.6$ & $35.6 \pm 7.6$ & $64.7 \pm 3.2$ \\
\hline & Control & $96.3 \pm 1.2$ & $3.7 \pm 1.2$ & 0.0 & $7.3 \pm 1.5$ \\
\hline \multirow{5}{*}{ Methanol } & 3.33 & $22.7 \pm 2.5$ & $77.3 \pm 2.5$ & $76.7 \pm 3.0$ & $136.0 \pm 3.6$ \\
\hline & 1.67 & $38.3 \pm 2.1$ & $61.7 \pm 2.1$ & $60.6 \pm 2.9$ & $130.0 \pm 3.5$ \\
\hline & 0.83 & $51.7 \pm 2.1$ & $48.3 \pm 2.1$ & $46.9 \pm 1.8$ & $109.0 \pm 7.0$ \\
\hline & 0.42 & $65.3 \pm 3.5$ & $34.7 \pm 3.5$ & $32.8 \pm 5.1$ & $91.7 \pm 4.0$ \\
\hline & Control & $79.3 \pm 2.1$ & $2.7 \pm 2.1$ & 0.0 & $7.3 \pm 3.5$ \\
\hline \multirow{5}{*}{$\begin{array}{l}\text { Ethyl Ace- } \\
\text { tate }\end{array}$} & 3.33 & $18.7 \pm 3.2$ & $81.3 \pm 3.2$ & $80.5 \pm 3.1$ & $125.7 \pm 4.7$ \\
\hline & 1.67 & $24.7 \pm 4.0$ & $75.3 \pm 4.0$ & $74.2 \pm 4.6$ & $112.7 \pm 4.7$ \\
\hline & 0.83 & $48.7 \pm 3.5$ & $51.3 \pm 3.5$ & $49.1 \pm 3.2$ & $101.0 \pm 4.4$ \\
\hline & 0.42 & $59.0 \pm 3.6$ & $41.0 \pm 3.6$ & $38.3 \pm 4.3$ & $90.3 \pm 0.6$ \\
\hline & Control & $95.7 \pm 1.5$ & $4.3 \pm 1.5$ & 0.0 & $6.7 \pm 2.1$ \\
\hline
\end{tabular}


Table 3: Repellent activity of $D$. triradiata different extracts against $C x$. antennatus.

\begin{tabular}{|c|c|c|c|c|c|}
\hline Extract & $\begin{array}{c}\text { Dose } \\
\left(\mathrm{mg} / \mathrm{cm}^{2}\right)\end{array}$ & $\begin{array}{l}\text { Fed females } \\
(\%)\end{array}$ & $\begin{array}{c}\text { Unfed females } \\
(\%)\end{array}$ & $\begin{array}{c}\text { Average repellency } \\
(\%)\end{array}$ & $\begin{array}{l}\text { Complete repellency time } \\
\text { (min.) }\end{array}$ \\
\hline \multirow{5}{*}{ Hexane } & 3.33 & $9.3 \pm 1.5$ & $90.7 \pm 1.5$ & $90.4 \pm 1.6$ & $158.0 \pm 7.9$ \\
\hline & 1.67 & $17.7 \pm 2.5$ & $82.3 \pm 2.5$ & $81.8 \pm 2.6$ & $146.3 \pm 6.1$ \\
\hline & 0.83 & $29.3 \pm 0.6$ & $70.7 \pm 0.6$ & $69.9 \pm 0.4$ & $136.0 \pm 9.8$ \\
\hline & 0.42 & $53.7 \pm 5.7$ & $46.3 \pm 5.7$ & $44.8 \pm 6.1$ & $112.7 \pm 5.7$ \\
\hline & Control & $97.3 \pm 0.6$ & $2.7 \pm 0.6$ & 0.0 & $6.7 \pm 2.3$ \\
\hline \multirow{5}{*}{ Chloroform } & 3.33 & $16.3 \pm 2.5$ & $83.7 \pm 2.5$ & $83.2 \pm 2.9$ & $175.3 \pm 2.1$ \\
\hline & 1.67 & $28.3 \pm 2.1$ & $71.7 \pm 2.1$ & $72.2 \pm 2.1$ & $155.3 \pm 4.2$ \\
\hline & 0.83 & $33.7 \pm 1.5$ & $66.3 \pm 1.5$ & $65.4 \pm 1.1$ & $141.0 \pm 3.6$ \\
\hline & 0.42 & $59.3 \pm 4.7$ & $40.7 \pm 4.7$ & $39.0 \pm 5.8$ & $121.7 \pm 3.2$ \\
\hline & Control & $97.3 \pm 1.5$ & $2.7 \pm 1.5$ & 0.0 & $7.7 \pm 1.2$ \\
\hline \multirow{5}{*}{ Methanol } & 3.33 & $16.3 \pm 4.0$ & $83.7 \pm 4.0$ & $82.7 \pm 4.2$ & $169.0 \pm 1.7$ \\
\hline & 1.67 & $31.7 \pm 3.5$ & $68.3 \pm 3.5$ & $66.4 \pm 4.2$ & $157.7 \pm 4.6$ \\
\hline & 0.83 & $49.0 \pm 2.6$ & $51.0 \pm 2.6$ & $48.0 \pm 3.6$ & $149.3 \pm 2.5$ \\
\hline & 0.42 & $58.3 \pm 4.2$ & $41.7 \pm 4.2$ & $38.1 \pm 5.1$ & $147.0 \pm 10.1$ \\
\hline & Control & $94.3 \pm 1.5$ & $5.7 \pm 1.5$ & 0.0 & $8.3 \pm 2.5$ \\
\hline \multirow{5}{*}{$\begin{array}{l}\text { Ethyl Ace- } \\
\text { tate }\end{array}$} & 3.33 & $10.7 \pm 4.0$ & $89.3 \pm 4.0$ & $89.0 \pm 4.0$ & $167.7 \pm 5.9$ \\
\hline & 1.67 & $20.3 \pm 2.5$ & $79.7 \pm 2.5$ & $79.1 \pm 2.5$ & $166.7 \pm 3.8$ \\
\hline & 0.83 & $36.7 \pm 4.9$ & $63.3 \pm 4.9$ & $62.3 \pm 4.5$ & $158.3 \pm 4.2$ \\
\hline & 0.42 & $42.3 \pm 2.1$ & $57.7 \pm 2.1$ & $56.4 \pm 1.3$ & $146.7 \pm 8.3$ \\
\hline & Control & $97.0 \pm 2.0$ & $3.0 \pm 2.0$ & 0.0 & $9.7 \pm 2.1$ \\
\hline
\end{tabular}

Table 4: $\mathrm{RD}_{50} \& \mathrm{RD}_{90}$ mean values of $D$. triradiata different extracts against mosquito strains used.

\begin{tabular}{|c|c|c|c|c|c|c|c|}
\hline \multirow{2}{*}{$\begin{array}{l}\text { Mosquito Spe- } \\
\text { cies }\end{array}$} & \multirow{2}{*}{$\begin{array}{l}\text { Extract } \\
\text { used }\end{array}$} & \multirow{2}{*}{$\begin{array}{c}\mathrm{RD}_{50} \\
\left(\mathrm{mg} / \mathrm{cm}^{2}\right)\end{array}$} & \multicolumn{2}{|c|}{ 95\% Confidence Limits } & \multirow{2}{*}{$\begin{array}{c}\mathrm{RD}_{90} \\
\left(\mathrm{mg} / \mathrm{cm}^{2}\right)\end{array}$} & \multicolumn{2}{|c|}{ 95\% Confidence Limits } \\
\hline & & & LCL & UCL & & LCL & UCL \\
\hline \multirow{4}{*}{$\begin{array}{c}\text { Anopheles ser- } \\
\text { gentii }\end{array}$} & Hexane & 0.704 & 0.4703 & 0.9377 & 2.567 & 2.423 & 2.710 \\
\hline & Chloroform & 1.101 & 0.8516 & 1.348 & 2.92 & 2.741 & 3.099 \\
\hline & Methanol & 1.183 & 1.105 & 1.225 & 3.067 & 2.923 & 3.210 \\
\hline & Ethyl Acetate & 0.904 & 0.8242 & 0.9832 & 2.88 & 2.579 & 3.181 \\
\hline \multirow{4}{*}{ Culex pipiens } & Hexane & 1.122 & 0.9513 & 1.282 & 3.027 & 2.883 & 3.170 \\
\hline & Chloroform & 1.367 & 1.249 & 1.484 & 3.317 & 3.058 & 3.575 \\
\hline & Methanol & 1.578 & 1.311 & 1.849 & 3.593 & 3.245 & 3.941 \\
\hline & Ethyl Acetate & 1.323 & 1.198 & 1.448 & 3.547 & 2.050 & 5.044 \\
\hline \multirow{4}{*}{ Culex antennatus } & Hexane & 0.92 & 0.8062 & 1.034 & 2.703 & 2.527 & 2.880 \\
\hline & Chloroform & 1.157 & 0.9312 & 1.382 & 3.09 & 2.773 & 3.407 \\
\hline & Methanol & 1.387 & 1.178 & 1.595 & 3.267 & 2.890 & 3.643 \\
\hline & Ethyl Acetate & 0.9 & 0.7060 & 1.094 & 2.81 & 2.508 & 3.112 \\
\hline
\end{tabular}

\section{Discussion}

Mosquito repellents are one of the most effective strategies in reducing the spread of diseases transmitted by different mosquito species. There are ongoing efforts in searching for a safer, better, and cheaper repellent agents against mosquito vectors, plant extracts providing a potential mosquito control agents, with low-cost, easy-to-administer, and risk-free properties. The present study showed that Deverra triradiata tested extracts displayed variable repellent activities against different mosquitoes (An. sergentii, $C x$. pipiens \& $C x$. antennatus) reflected the complexity of the chemical composition of their constituents (Bisseleua et al, 2008). The repellent effect of tested extracts may be due to the presence of various compounds, including phenolics, terpenoids and alkaloids, which exist in D. triradiata; these compounds may jointly or independently contribute to produce a repellent activity Rajkumar and Jebanesan, (2005). Also, the repellent activity varied according to solvent used in extraction and the dose of the extract, as hexane extract was more effective in exhibiting the repellent action against three tested mosquito species than chloroform, methanol and ethyl acetate extracts. The present repellent activity exhibited by 
D. triradiata extracts agreed with results of Yang et al, (2004), where methanolic extract of Cinnamomum cassia (bark), Nardostachys chinensis (rhizome), Paeonia suffruticosa (root bark) and Cinnamomum camphora gave 91.0, 81.0, $80.0 \& 94.0 \%$ repellent activities against starved Ae. aegypti at $0.1 \mathrm{mg} /$ $\mathrm{cm}^{2}$, Mullai et al. (2008) using benzene, petroleum ether, ethyl acetate \& methanol extracts of Citrullus vulgaris leaf for An. stephensi, Govnidarajan and Sivakumar (2011) using crude hexane, ethyl acetate, benzene, chloroform and methanol extracts of Eclipta alba and Andrographis paniculata leaf against $A$ e. aegypti at $1.0,2.5, \& 5.0 \mathrm{mg} / \mathrm{cm}^{2}$ and they suggested that the leaf solvent plant extracts have the potential to be used as an ideal eco-friendly approach for the mosquitoes control and El-Sheikh et al. (2012) using methanolic extract of Tribulus terrestris (leaves \& seeds) against An. arabiensis, where the seeds extract recorded $100 \%$ repellent action at $1.0 \mathrm{mg} / \mathrm{cm}^{2}$ against females compared with $79.5 \%$ repellent activity caused by leaves extract at $2.0 \mathrm{mg} / \mathrm{cm}^{2}$.

Similar results were recorded by Hassan $e t$ $a l$, (2014) for ethanol, acetone and petroleum ether extracts from Lagenaria siceraria (leaves $\&$ stems) against $C x$. pipiens, Sabiha et al. (2017) for petroleum ether, chloroform and methanol extracts of Melia azedarach leaf which offered repellent activity at 5\% level of significance $(P<0.05)$ against $C x$. quinquefasciatus and Bream et al. (2018) for ethanol $70 \%$, acetone, chloroform and petroleum ether extracts from Musca acuminata leaves which evoked a variable degree of repellency against $C x$. pipiens starved females.

In the present study, the tested extracts showed a strong biting deterrency against tested mosquito species according to solvent used in extraction. In general, the tested extracts provided a complete protection time ranging from 57.7 to $187.7 \mathrm{~min}$ against $A n$. sergentii, $C x$. pipiens and $C x$. antennatus bites, which agreed with Venkatachalam and Jebanesan (2001) who used methanol extract of Fredonia elephantum leaves against $A e$. aegypti at $1.0 \& 2.5 \mathrm{mg} / \mathrm{cm}^{2}$ concentrations and reported $100.0 \%$ protection up to $2.14 \&$ 4.0 h, Rajkumar and Jebanesan (2004) used Moschosma polystachyum crude leaf extract showed $85.2 \& 54.6 \mathrm{~min}$ protection against Cx. quinquefasciatus bites at $1.0 \& 2.5 \mathrm{mg} /$ $\mathrm{cm}^{2}$, Rajkumar and Jebanesan (2005) using volatile oils extracted from leaves of Moschosma polystachyum \& Solanum xanthocarpum against $C x$. quinquefasciatus, where the oil from $M$. polystachyum \& S. xanthocarpum gave $332.2 \& 311.4 \mathrm{~min}$ protection against mosquito bites at $4 \& 8 \%$ vs. $4.4 \mathrm{~min}$ protection in controls. The volatile oils of these two plant species were effective as repellents and gave more than 300min $(>5$ hour) protection against $C x$. quinquefasciatus bite. Mullai et al, (2008) found that benzene, petroleum ether, ethyl acetate and methanol extracts of $C x$. vulgaris (leaf) at $1.0,2.5 \& 5.0 \mathrm{mg} / \mathrm{cm}^{2}$ gave mean complete protection time against An. stephensi ranged from 119.17 to $387.83 \mathrm{~min}$. Adhikari and Chandra (2014) found that petroleum ether leaf extract of Swietenia mahagoni showed repellency up to $2 \mathrm{~h}$ against $A n$. stephensi.

\section{Conclusion}

Deverra triradiata extracts proved to have a good repellent activity against Anopheles sergentii, Culex pipiens and Culex antennatus. Extensive studies are ongoing to identify the bioactive compound(s) responsible for repellent activity to be prepared as commercial product /formulation.

\section{References}

Abbott, WS, 1925: A method for computing the effectiveness of an insecticide. J. Econ. Entomol. 18: 265-77.

Abdel-Motagaly, AME, Mohamad, HM, Morsy, TA, 2017: A mini-re-view on skeeter syndrome or large local allergy to mosquito bites. J. Egypt. Soc. Parasitol. 47, 2:415-24.

Adhikari, U, Chandra, G, 2014: Larvicidal, smoke toxicity, repellency and adult emergence inhibition effects of leaf extracts of Swietenia mahagoni Linnaeus against Anopheles stephensi Liston (Diptera: Culicidae). Asian Pac. J. Trop. Dis. 4, 1:279-83.

Bisseleua, HBD, Gbewonyo, SWK, Obeng-Of- 
ori, D, 2008: Toxicity, growth regulatory and repellent activities of medicinal plant extracts on Musca domestica L. (Diptera: Muscidae). Afr. J. Biot. 7, 24: 4635-42.

Bream, AS, Shehata, AZI, Zaki, MSM, 2018: Biological activity of Musca acuminata (Musaceae) extracts against the mosquito vector, Culex pipiens L. (Diptera: Culicidae). J. Egypt. Soc. Parasitol. 48, 2:261-70.

El-Bahnasawy, MM, Abdel Fadil, EE, Morsy, TA, 2013: Mosquito vectors of infectious diseases: Are they neglected health disaster in Egypt? J. Egypt. Soc. Parasitol. 43, 2: 373-86

El-Sheikh, TMY, Al-Fifi, ZIA, Alabboud, M A, 2016: Larvicidal and repellent effect of some Tribulus terrestris L., (Zygophyllaceae) extracts against the dengue fever mosquito, Aedes aegypti (Diptera: Culicidae). J. Saudi Chem .Soc. 20:13-9.

El-Sheikh, TM, Bosly, HM, Shalaby, N, 2012: Insecticidal and repellent activities of methanolic extract of Tribulus terrestris L. (Zygophyllaceae) against the malarial vector Anopheles arabiensis (Diptera: Culicidae). Egypt. Acad. J. Biol. Sci. 5, 2:13-22.

Govnidarajan, M, Sivakumar, R, 2011: Adulticidal and repellent properties of indigenous plant extracts against Culex quinquefasciatus and Aedes aegypti (Diptera: Culicidae). Parasitol. Res. 109, 2:353-67.

Haldar, KM, Ghosh, P, Chandra, G, 2014: Larvicidal, adulticidal, repellency and smoke toxic efficacy of Ficus krishnae against Anopheles stephensi Liston and Culex vishnui group mosquitoes. Asian Pac. J. Trop. Dis. 4, 1:214-20.

Hanafi, HA, Fryauff, DJ, Saad, MD, Soliman, AK, Mohareb, EW, et al, 2011: Virus isolations and high population density implicate $\mathrm{Cu}$ lex antennatus (Becker) (Diptera: Culicidae) as a vector of Rift Valley Fever virus during an outbreak in the Nile Delta of Egypt. Acta Trop. 119, 2/3:119-24

Hassan, MI, Fouda MA, Hammad, KM, Tanani, MA, Shehata, AZ, 2014: Repellent effect of Lagenaria siceraria extracts against Culex pipiens. J. Egypt. Soc. Parasitol. 44, 1:243-48.

Mikhail, MW, Al-Bursheed, KhM, Abdel-Halim, AS, Morsy, TA, 2009: Studies on mosquito borne diseases in Egypt and Qatar. J. Egypt. Soc. Parasitol. 39, 3:745-56.

Morsy, TA, 2018: Aedes aegypti and dengue virus infections. J. Egypt. Soc. Parasitol. 48, 1: 183-96.
Mullai, K, Jebanesan, A, Pushpanathan, T, 2008: Mosquitocidal and repellent activity of the leaf extract of Citrullus vulgaris (cucurbitaceae) against the malarial vector, Anopheles stephensi Liston (Diptera: Culicidae). Eur. Rev. Med. Pharmacol. Sci. 12:1-7.

Pitasawat, B, Choochote, W, Tuetun, B, Tippawangkosal, $\mathrm{P}$, Kanjanapothi, D, et al, 2003: Repellency of aromatic turmeric Curcuma aromatica under laboratory and field conditions. J. Vector Ecol. 28, 2:234-40.

Qui, H, Jun, HW, McCall, JW, 1998: Pharmacokinetics, formulation, and safety of insect rep ellent N, N-diethyl-3-methylbenzamide (DEET): A review. J. Am. Mosq. Control Assoc. 14:1227.

Rajkumar, S, Jebanesan, A, 2004: Mosquitocidal activities of octacosane from Moschosma polysta-chyum Linn (Lamiaceae). J. Ethnopharmacol. 90, 1:87-9.

Rajkumar S1, Jebanesan A, 2005: Repellency of volatile oils from Moschosma polystachyum and Solanum xanthocarpum against filarial vector Culex quinquefasciatus Say. Trop. Biomed. 22, 2:139-42.

Sabiha, S, Ali, H, Hasan, K, Rahman, ASMS, Islam, N, 2017: Bioactive potentials of Melia azedarach $\mathrm{L}$. with special reference to insecticidal, larvicidal and insect repellent activities. J. Entomol. Zool. Stud/ 5, 5:1799-802.

SPSS, 2007: SPSS for windows. Version 11.5, Chicago, IL, USA.

Tawatsin, A, Wratten, SD, Scott, RR, Thavara, U, Techadamrongsin, Y, 2001: Repellency of volatile oils from plants against three mosquito vectors. J. Vector Ecol. 26, 1:76-82.

Venkatachalam, MR, Jebanesan, A, 2001: Repellent activity of Ferronia elephantum Corr. (Rutaceae) leaf extract against Ae. aegypti L. Bioresour. Technol. 76, 3:287-8.

Walker, TW, Robert, LL, Copeland, RA, Githeko, AK, Wirtz, RA, et al, 1996: Field evaluation of arthropod repellents, DEET and a piperidine compound, A13-37220, against Anopheles funestus and Anopheles arabiensis in Wes-tern Kenya. J. Am. Mosq. Control Assoc. 12: 172-6. Yang, YC, Lee, EH, Lee, HS, Lee, DK, Ahn, YG, 2004: Repellency of aromatic medicinal plant extracts and a steam distillate to Ae. aegypti. J. Am. Mosq. Control Assoc. 20, 2:146-9. WHO, 2014: World Malaria Report. Available from: http://www.who.int/iris/bitstream/10665/ 97008/1/9789241564694_eng.pdf. 